\title{
St. Petersburger Ophthalmologische Gesellschaft
}

\section{Sitzung am 23. April und 14. Mai 1909}

Demonstriert wird von Kul·lí eine 23 jährige Patientin, die am 3. IV. etwa 10 g Chinin genommen hatte, wonach Blindheit eintrat, die allmählich

$25^{*}$

372 Unfall- und Versicherungskunde.

zurückging, und heute besteht fast voile zentrale Sehschärfe, aber das Ge-sichtsfeld ist bis auf wenige Grade um den Fixationspunkt eingeschränkt, und die Papillen sind blass, die Gefässe eng, kurz das Bild einer genuinen Sehnervenatrophie nach Chininvergiftung.

Dr. Weinstein demonstriert mikroskopische Präparate der kongeni-talen serösen orbito palpebralen Cyste (cfr. Bericht der Sitzung am 11. XII. 1908). In diesem Falle war neben der Cyste ein normaler Aug-apfel vorhanden. Das Präparat zeigt eine einfache seröse Cyste, die in ihrem Bau gar keine Aehnlichkeit zeigt mit den Cysten, die in der Orbita gefunden werden bei Fehlen des Augapfels oder bei Mikrophthalmos congenitus.

Kubli berichtet kurz über den Verlauf des XI. internationalen ophthal-mologischen Kongresses in Neapel vom 2.-7. IV. 1909. Für den nächsten, im Jahre 1914 abzuhaltenden Kongress ist unsere Einladung angenommen worden, und wird der XII. Kongress voraussichtlich Ende Juli alten Stils nach St. Petersburg berufen werden.

Dr. Blessig: Ein Fall von Retino-Chorioiditis juxta papillaris (Edmund Jensen, Gr. Arch. 69, 1). Krankengeschichte und Bilder. In der Anamnese weder Lues noch Tuberkulose.

Dr. Tschemolosof: Ueber praktische Resultate, erzielt mit Hülfe der Unterweisung intelligenter Nichtmediziner im Erkennen und Behandeln des Traehoms.

Dr. Hulanicki hat einen kurzen Bericht zur Verlesung eingesandt. Er hat in Welikoluzk 15

Personen unterwiesen. Bei reichem Material halt er es für möglich, 6 Personen in 6 bis 8 Wochen so weit zu fördern, dass sie die Trachomkrankheit in alien Stadien richtig erkennen, die eГste Hülfe richtig leisten können und eine Weiterbehandl·ung nach $\Lambda \tau$ orschrift des Arztes $\mathrm{dl} \cdot$ irchzuführen verstehen. Zwei Lehrerinnen hat er so $\tau$ veit vorgebildet Die anderen arbeiteten zu kurze Zeit, um so weit zu kommen, von alien darf aber erwartet werden, da $\approx \mathrm{s}$ sie die Kranken belehren und zugleich veranlassen werden, Hülfe aufzusuchen.

Tschemolosof hat mit 12 Personen gearbeitet und ebenfalls nicht alle gleich ausbilden können, ist aber mit den erzielten Resultaten durchaus zufrieden und wünscht, unsere jährlich ansgesandten sogenannten fliegenden augenärztlichen Kolonnen sollen diese Tätigkeit mit in ihr Programm auf-nehmen. Es ist freilich weiterhin nötig, nach Jahr und Tag zu kontrollieren, was diese Unterwiesenen mit ihren Kenntnissen praktisch erreicht und ge-leistet haben.

Sitzung am 14. Mai 1909.

Dr. Tschemolosof: Nachruf für den am 20. IV. zu Dorpat verschiedenen Professor Th. Ewetzlci. W. Kalaschniko $\dagger$ demonstriert eine Patientin mit in die vordere Kammer luxierter Linse. Wie die Luxation zustande gekommen ist, kann nicht sicher festgestellt werden, da ein Trauma oder Fall nicht zugegeben wird. Weiter demonstriert er eine Patientin mit Embolie der Netzhautarterie und 
Insuffi-cientia valvulae bicuspidalis. Zur Zeit ist der Fall bereits in Rückbildung und Besserung begriffen.

A. Filipof: Ueber Glashautbildung in der vorderen Augenkammer. Demonstration von Präparaten.

A. Weinstein berichtet über einen Fall von Sehnervenatrophie naeh subkutaner Anwendung von Arsaeetin bei Febris recurrens. Der Mann hat 2 Einspritzungen zu 0,5 und 0,7 g erhalten

Germann.

Unfall- und Versicherungskunde.

D. Die gesetzliche Unfallversieherung erstreckt sich auf alle Gefaiiren

die durch den Betrieb geboten werden. Hierzu gehören auch die „Ge-fahren des täglichen Lebens"

sofern der Versicherte ihnen infolge seiner Betriebstätigkeit ausgesetzt ist. Rekursentscheidung 2305 des 